\title{
Physical features vs meaning: A difference in decay*
}

\author{
LARRY L. JACOBY $\dagger$ \\ Iowa State University, Ames, Iowa 50010
}

\begin{abstract}
The encoding of either physical or semantic features of words was biased in an intentional learning situation. A modified recognition test was then employed to assess the effectiveness of this study manipulation and its consequences for retention. The $\mathrm{Ss}$ were required to select test items that were either physically similar, semantically similar, or identical to a study word. Results revealed that Ss biased toward physical encoding were more successful in selecting physically similar than semantically similar test items, while the opposite was true of Ss biased toward semantic encoding. The Ss in the two study conditions did not differ in their ability to select test items that were identical to a study word. This pattern of results was interpreted as evidence that semantic and physical information can be equally well retained over the long term. Limits on the generality of prior findings of rapid decay for physical information are discussed.
\end{abstract}

The memory trace can be characterized as a collection of attributes representing physical, semantic, and other features of a presented item (Underwood, 1969; Wickens, 1970). This characterization gains considerable power if attributes can be shown to differ in details surrounding their construction or retention. One possibility is that the physical features of an item, such as its sound or visual appearance, are forgotten more rapidly than are its semantic features. Although several experiments have shown rapid forgetting of physical features, the generality of this result is questionable. For exampie, the ability to recognize a friend's voice appears to demonstrate long-term retention of physical features. The present experiment was an attempt to demonstrate that physical features can be retained over the long term. A procedure for emphasizing either physical or semantic features during study was employed, and the encoding effects of this procedure were then assessed along with its consequences for retention.

Most experiments showing poor retention of physical information have used incidental learning procedures (e.g., Craik, 1973; Elias \& Perfetti, 1973; Jacoby \& Goolkasian, 1973). For example, Ss might be required to generate either rhymes or associates for each list item, and then an unexpected retention test is given. The greater retention when Ss are required to engage in a semantic task has led to the conclusion that semantic information decays less rapidly than physical information. However, the possiblity exists that the disadvantage of physical information observed in these experiments is due to to the particular tasks used to bias encoding. Physical and semantic information might be equally well retained if encoding was biased by other means.

*This article was prepared while the author was on leave, visiting the University of Toronto. The author expresses his appreciation to F. I. M. Craik, Zita M. Simutis, and a consulting editor who remains anonymous for their helpful comments concerned with the writing of this paper.

tRequests for reprints should be sent to Larry L. Jacoby, Department of Psychology. Iowa State University, Ames, Iowa 50010.
One means of biasing study encoding in an intentional learning situation would be to manipulate the study context surrounding an item. For example, the physical features of the word "dog" would be emphasized if it followed "log" in a study list. In contrast, the semantic features of $\operatorname{dog}$ would be emphasized if dog was presented after cat. Presenting a list containing words that were physically similar or a list containing words that were semantically similar might also result in a "list set"; Ss would expect a particular type of similarity and would modify their encoding of list items accordingly. If so, the encoding of unrelated words embedded in a list would be biased toward the class of attributes underlying the similarity between related items.

Employing the above rationale, study lists in the present experiment were constructed by randomly arranging an equal number of related and unrelated pairs of words, with members of a pair presented successively. Two study lists differed in that one contained pairs of words that were semantically similar, while the other contained pairs of words that were physically similar. Items in unrelated pairs were common to the two study lists, but their encoding was expected to differ between lists due to the influence of the list set created by the related pairs. For example, presenting pairs of semantically similar words was expected to result in semantic encoding of words presented in unrelated pairs.

Before conclusions can be drawn about differences in the decay of physical and semantic information, evidence that is independent of overall differences in retention must be provided to show that study encoding has been biased. One means of demonstrating differences in encoding has made use of false recognition errors (Elias \& Perfetti, 1973). The assumption underlying the false recognition procedure is that the attractiveness of a new test item depends on its similarity to a study encoding. For example, a false recognition of a test item that is semantically similar to a study item is taken as evidence that the study item was encoded semantically. A difficulty with this technique is that false recognitions 
Table 1

Example Study and Test Pairs

\begin{tabular}{|c|c|c|c|}
\hline \multicolumn{2}{|c|}{ Study Lists } & \multirow{2}{*}{$\begin{array}{l}\text { Test } \\
\text { Pair }\end{array}$} & \multirow[b]{2}{*}{ Study-Test Relationship } \\
\hline Semantic & Physical & & \\
\hline $\begin{array}{l}\text { Arm } \\
\text { Leg }\end{array}$ & $\begin{array}{l}\text { Beg } \\
\text { Leg }\end{array}$ & $\begin{array}{l}\text { Debt } \\
\text { Leg }\end{array}$ & Related Pair/Identical \\
\hline \multirow[t]{2}{*}{$\begin{array}{l}\text { Football } \\
\text { Book }\end{array}$} & $\begin{array}{l}\text { Football } \\
\text { Book }\end{array}$ & $\begin{array}{l}\text { Football } \\
\text { Bell }\end{array}$ & Unrelated Pair/Identical \\
\hline & & $\begin{array}{l}\text { Motor } \\
\text { Cook }\end{array}$ & Physically Similar \\
\hline $\begin{array}{l}\text { House } \\
\text { Verb }\end{array}$ & $\begin{array}{l}\text { House } \\
\text { Verb }\end{array}$ & $\begin{array}{l}\text { Truth } \\
\text { Noun }\end{array}$ & Semantically Similar \\
\hline
\end{tabular}

Note-The italicized item in test pairs is the correct response.

are often few in number. Furthermore, the absence of a false recognition provides no information about study encoding. False recognitions can fail to occur either because attributes of a particular class (for example, semantic attributes) were not encoded during study or because encoding of the attributes was complete enough to allow discrimination between the memory trace of a study item and the encoding of a similar test item.

A method of avoiding problems associated with the false recognition procedure is to apply the same underlying rationale to a situation in which $\mathrm{Ss}$ are instructed to select test items that are either similar or identical to a study item. The Ss' success in selecting test items that are similar to a study item will reflect the way the study item has been encoded; furthermore, this procedure will have the advantage of relying on correct responses rather than errors to provide usable data. The discriminability of study and test encodings can also be measured by further instructing Ss to indicate whether selected items are identical or only similar to a study item. The ability to identify a test item as being similar requires a discriminable difference between the memory trace of the study item and the encoding of a similar test item. Selection of a similar test item and misidentification of that item as being identical to a study word corresponds to a false recognition error.

The construction of study lists and the test procedure will be further described and illustrated with examples in the Method section.

\section{METHOD}

\section{Rationale}

Since the argument presented is a little complex, the rationale of the experiment will be spelled out before describing the study in detail. Two groups of Ss each received a 60-word list to learn; the list was presented as 30 word pairs. For one group of Ss, the list contained 15 semantically related pairs (e.g., ARM LEG) plus 15 unrelated pairs (e.g., FOOTBALL BOOK), while the list for the other group contained 15 physically related pairs (e.g., BEG LEG) plus the same 15 unrelated pairs. The related and unrelated pairs were randomly mixed within the list. It was expected that the "semantic" group would quickly learn to process all words semantically and that the "physical" group would process words in terms of their physical characteristics.
Example pairs from the two study lists and example test pairs are shown in Table 1 .

As illustrated by the test pair in Table 1 , the Ss were to select items that were either similar or identical to a study word. The results were analyzed in two steps. First, differential processing between the two study groups was checked by analyzing differences in the selection of words that were similar to a word in the presentation list. If the two groups of Ss did indeed process words differently, it would be expected that the semantic group would be better able to select test items that were semantically similar to a study word, while the physical group would be better able to select items that were physically similar to a study word. Assuming that this analysis yielded evidence for encoding bias, the second step was to examine the recognition of words that were identical to a word in the presentation list. If semantic encoding yields superior memory performance, then recognition should be higher for the semantic study condition.

\section{Materials}

Pairs of semantically related words were constructed by choosing two of the five most frequent instances from each of 15 categories listed in the Battig and Montague (1969) norms. Pairs of physically related words were then constructed by replacing one member of each semantically related pair with a word that rhymed with the remaining member; the first member and the second member of semantically related pairs was replaced equally often. In all cases, members of a physically related pair were common words that differed only in the initial syllable, so physically related words were similar in both spelling and sound. The 15 unrelated pairs contained words chosen from 30 remaining categories listed in the Battig and Montague norms; one word was selected from the five most frequent instances of each category. In selecting words for unrelated pairs, an attempt was made to minimize semantic and physical similarity both within and between pairs. The order of related and unrelated pairs was randomly determined.

Recognition test pairs were constructed from 40 words that were common to the two study lists and from 40 new words that were selected to be unrelated to any study word. One item in each test pair was either similar or identical to a study word; the other item in each test pair was a new unrelated word. Example test pairs are presented in the third column of Table 1. The last column of Table 1 describes the relationship between a study and test item that is exemplified by the test pairs.

Of the 40 test pairs, 20 included an item that was identical to a word contained in the study lists; 10 of these items were drawn from "related" study pairs, while the remaining 10 items were drawn from "unrelated" study pairs. Related study pairs provided only test items that were identical to a study word, and only one member of any related study pair was represented at test. The remaining 20 test pairs contained an item that was similar to a member of an unrelated study pair. Ten of the target items in these test pairs were from the same category as a study word and among the five most frequent instances of that category in the Battig and Montague norms. The 10 remaining target items were similar in sound and spelling to a member of an unrelated study pair; these physically similar test items differed from a study item only in the initial syllable.

Within each of the four types of target items (identical-related study pair, identical-unrelated study pair, semantically similar, and physically similar), there was an equal division of items representing a word in the first and a word in the second position of a study pair. Dissimilar items in each of the 40 test pairs were common words that were chosen to be both physically and semantically dissimilar to any study itern. Four random orders of test pairs were constructed; the position of the target item within a test pair was randomly assigned for each of the orders. 


\section{Procedure}

Study words were prepared as slides and presented individually at a 3 -sec rate, with members of a study pair presented successively. Prior to presentation of the study list, Ss were informed that the study list was composed of successively presented word pairs; the nature of the relationships contained in the list was also specified. The Ss were told they would be tested for their retention of the list, but the nature of the test was not specified.

After list presentation, all Ss were read five sets of nine digits, formed by randomly arranging the digits $0-9$, and asked to recall each set immediately after its presentation. The purpose of the digit recall task was to eliminate any short-term memory of list words. The digit recall task took approximately $2 \mathrm{~min}$.

After the digit recall task, Ss were presented with a test deck consisting of 40 note cards; each note card contained a single test pair. The Ss were informed that one member of each test pair was either acoustically similar, semantically similar, or identical to a study word. They were then instructed to select the appropriate item from each test pair, indicate the similarity that existed between the selected item and a study word, and rate their confidence in their combined responses on a 99-point scale.

Ss recorded their responses on a mimeographed sheet of paper. The selected item from each test pair was written in a numbered blank corresponding to the number of the test card. Next, Ss circled either I (identical), S (semantic), or A (acoustic) to indicate the similarity they felt existed between the selected item and a study word; confidence judgments were recorded in a blank that was adjacent to the similarity designations. There was no time limit on the recognition test.

\section{Subjects}

The Ss were 24 students enrolled in psychology courses at Iowa State University, who participated in the experiment for course credit. Twelve Ss were assigned to each of the two study conditions, viewing either a list containing semantically related pairs or a list containing physically related pairs. The Ss were tested in small groups that ranged in size from one to four; all Ss tested in a given session viewed the same study list. The assignment of incoming groups to experimental conditions was random.

\section{RESULTS AND DISCUSSION}

Results from similar test items were used to determine if the study manipulation biased encoding. This is possible because selection of a test item that is similar to a study item requires that the at tribute serving as a basis of similarity was encoded during study. After reporting results from similar test items, the effects of study processing on recognition of test items that were identical to a study word will be described. The identical test items could be recognized on the basis of either semantic or physical attributes encoded during study. This being the case, differences in recognizing test items that are identical to a study word should reflect any retention advantage of semantic encoding. The Ss in the condition biased toward semantic encoding should be more successful in recognizing identical test items if physical information is less likely to be retained over the long term.
Table 2

Probabilities of Selecting Similar Test Items

\begin{tabular}{ccc}
\hline & \multicolumn{2}{c}{ Similarity of Test Item } \\
\cline { 2 - 3 } Study & Physical & Semantic \\
\hline Condition & .66 & .55 \\
Physical & .59 & .73 \\
\hline
\end{tabular}

\section{Similar Test Items}

All similar test items were similar only to a member of an unrelated study pair shared by the two study conditions. Thus, any effects of study condition must be entirely due to a "list set" created by including semantically or physically related items in the study list. The encoding effect of the study manipulation was assessed by analyzing Ss' success in selecting similar test items, regardless of whether the selected items were correctly identified as being similar. Once selected, success in identifying a test item as being semantically or physically similar to a study word was used as a measure of the discriminability of study and test encodings. Analyses of confidence judgment results will not be reported, since they did not provide any additional information.

Selection of Similar Test Items. The probabilities of selecting physically similar and semantically similar test items are shown in Table 2. In line with expectations, Ss in the semantic study condition were more successful in selecting test items that were semantically similar to a study word, while Ss in the physical study condition were more successful in selecting test items that were physically similar to a study word. The interaction of Study Condition by Similarity of Test Items was highly significant $[F(1,22)=16.39, p<.001]$. (The estimate of error variance used to test this interaction was .19.)

As Table 2 shows, the study encoding of any particular item was influenced by the relationships among other items included in the same study list. It should also be noted that, when the study bias and the similarity of test items was not in agreement, the probability of selecting a similar test item was near the chance level of $.50(.55, .59)$. That is, there was little evidence of encoding on the dimension that was contrary to that emphasized by the list set.

Identification of Similar Test Items. Probabilities of selecting a similar test item and correctly identifying the basis of similarity held by that test item and a study item are presented in Table 3 . The interaction of True and Judged Similarity $[F(2,44)=39.72, p<.001]$ was significant in revealing the similarity of selected test items was correctly identified in most cases. In general, if Ss were able to select a similar test item, they were also able to identify the similarity that existed between that test item and a study word. Although the relevant data are not shown in Table 3 , this ability to correctly identify the similarity of selected test items was 
Table 3

Judged Similarity as a Function of True Similarity

\begin{tabular}{lccc} 
& \multicolumn{3}{c}{ Judged Similarity } \\
\cline { 2 - 4 } Similarity & Identical & Physical & Semantic \\
\hline Physical & .08 & .41 & .13 \\
Semantic & .10 & .08 & .45 \\
\hline
\end{tabular}

Note-The estimated error variance used for testing the interaction of true and judged similarity was 1.58 .

uninfluenced by whether study was biased toward physical or semantic encoding.

\section{Identical Test Items}

Selection of Identical Test Items. The analysis of results from similar test items provided evidence that the presence of related words biased the encoding of other words in the study list. Since Ss were unable to predict how an item would be tested, the study manipulation must have biased the encoding of study items that were tested by means of an identical test item as well as those tested by means of a similar test item. If physical information decays faster than semantic information, the probability of selecting test items that are identical to a study word should be lower in the condition that was biased toward physical encoding during study.

Table 4 presents probabilities of successfully selecting a test item that was identical to a study word.

The selection of identical test items was not significantly influenced by whether the test item had originated from a related study pair or an unrelated study pair. Of greater importance, the probability of selecting an identical test item was slightly higher in the physical (.83) than in the semantic (.80) study condition. Athough this difference between encoding conditions did not approach significance, the numerical difference is opposite to that predicted by faster decay of physical information.

On the basis of the combined results from selection of similar and identical test items, the study manipulation biased encoding but resulted in equal recognition based on different memory attributes. Physical information was retained over the long term and held no disadvantage to semantic information.

Identification of Identical Test Items. As was done with similar test items, the probability of selecting and identifying an identical test item was used as a measure of the discriminability of study and test encodings. However, now a failure to correctly identify a selected item as being identical to a study word indicates a difference in the encoding of two nominally identical items. That is, study and test encodings of the same item are so different that they appear to the $S$ as being based on words that are only similar.

Probabilities of selecting an identical test item and identifying it as being identical, semantically similar, or physically similar to a study word are shown in Table 5 . The results in Table 5 are collapsed across study conditions (physical or semantic study bias) since interactions with study condition were not significant.

Identical test items are most often correctly identified as being identical to a study word $[\mathrm{F}(2,44)=126.74$, $\mathrm{p}<.001]$. However, the probability of selecting a test item and correctly identifying it was higher for test words originating from "unrelated" study pairs than for words originating from "related" study pairs $[F(2,44)=29.91, p<.001]$. This latter effect arose even though Ss were not significantly more successful in selecting test items that had been studied in unrelated pairs $(.78$ vs .84$)$. Thus, a test item from a related study pair was more likely to be misidentified as being only physically or semantically similar to a study word. In addition, though the data are not presented, there was a nonsignificant tendency toward incorrect identifications coinciding with study conditon. For example, a test item that had occurred in a semantically related study pair was more likely to be misidentified as being semantically similar (.12) rather than physically similar (.06) to a study word.

Differences in identification of items from related and unrelated pairs can be interpreted as being due to local context effects. Although a list set would influence the encoding of items in both related and unrelated study pairs, local context effects should be greater for items studied as a member of a related pair. Items in a related pair are likely to interact during study, so that the encoding of each reflects the influence of the other. Presenting a member of a related study pair with a new dissimilar item would then represent a more effective change between study and test contexts than would result for items studied in unrelated pairs. Due to the

Table 4

Probabilities of Selecting Identical Test Items

\begin{tabular}{|c|c|c|c|c|}
\hline \multirow[b]{2}{*}{$\begin{array}{c}\text { Study } \\
\text { Condition } \\
\end{array}$} & \multicolumn{4}{|c|}{ Origin of Test Item } \\
\hline & \multicolumn{2}{|c|}{$\begin{array}{c}\text { Related } \\
\text { Pair }\end{array}$} & $\begin{array}{l}\text { Unrelated } \\
\text { Pair }\end{array}$ & Mean \\
\hline Physical & \multicolumn{2}{|l|}{.80} & .86 & .83 \\
\hline Semantic & \multicolumn{2}{|l|}{.77} & \multirow{2}{*}{$\begin{array}{l}.83 \\
.84\end{array}$} & .80 \\
\hline Mean & \multicolumn{2}{|l|}{.78} & & .81 \\
\hline \multirow{3}{*}{ Judged Si } & \multicolumn{3}{|c|}{ Table 5} & \multirow{2}{*}{ from } \\
\hline & and Un & related $\mathbf{S}$ & tudy Pairs & \\
\hline & \multicolumn{4}{|c|}{ Judged Similarity } \\
\hline Study Pair & Identical & Physical & Semantic & Sum \\
\hline Related & .52 & .13 & .13 & .78 \\
\hline Unrelated & .75 & .01 & .08 & .84 \\
\hline
\end{tabular}

Note-The estimated error variances used for testing the main effect of judged similarity and the Study Pair by Judged Similarity interaction were 1.92 and .63 . 
large change in local context, a test item originating from a related study pair appears to the $S$ as being only similar to a study word.

\section{Some Theoretical Implications}

Results of the present experiment allow three main points to be made:

First, Ss are aware of which attributes overlap study and test encodings. The Ss were quite accurate in identifying test items as being physically or semantically similar to a study item. It is difficult to see how this result could be incorporated into a "strength" or "frequency" theory of recognition memory (e.g., Ekstrand, Wallace, \& Underwood, 1966). Rather than responding to the overall "strength" of a test item, Ss had information concerning the status of individual attributes.

The second point is that contact with the memory trace of a study item is not all-or-none. Prior recogntion effects of local context have been interpreted as evidence that retrieval processes are involved in recognition (e.g., Tulving \& Thomson, 1971). A failure in retrieval due to a change in context seems to imply that Ss were unable to gain contact with an existing memory trace that matched the test encoding. However, in the present experiment, success in selecting identical test items was approximately equal regardless of whether the items originated from related or unrelated study pairs. Thus, the probability of making trace contact was not drastically reduced by increasing the change in context. Rather, effects of context were primarily on the Ss' ability to identify a test item as being identical to a study item. This result suggests that similarity of context influences the extent of overlap between study and test encodings. A failure to recognize a test word as being identical to a study word would then be a result of a partial mismatch between study and test encodings. In a more extreme case (e.g., Light \& Carter-Sobell, 1970), there may be no overlap between study and test encodings of a nominally identical item, producing a complete failure to recognize.

The final point is that physical information can be retained over the long term as well as semantic information. Study encoding was manipulated to emphasize either physical or semantic attributes without influencing recognition performance. Prior experiments have found rapid decay of physical information by employing incidental learning procedures (Craik, 1973; Jacoby \& Goolkasian, 1973) or presenting sentences for study (Begg \& Paivio, 1969; Sachs, 1967). It is undoubtedly the case that in normal circumstances the word is treated as a symbol and processed only to the extent that is necessary to reach that which it symbolizes. However, with an appropriate set, the physical information embodied in a word can become the object of study and be remembered over the long term. Results of other experiments (Kolers, 1973;
Walter, 1973) agree with the present experiment in showing long-term retention of physical information. Since either semantic or physical information can be retained over the long term, there must be some factor other than the class of attributes chosen for encoding that determines retention.

It is quite unlikely that intentional learning procedures such as those employed in the present experiment are necessary to produce long-term retention of physical information. Instructions to learn must necessarily be translated into some form of processing, so that it is the processing and not the intention to learn that is essential. This suggests that incidental learning tasks could be constructed to mimic the results obtained in the present experiment. If so, the separation of incidental tasks into those that emphasize semantic properties and those that emphasize physical properties is too crude a division; research emphasis should be placed on the fine grain requirements of incidental learning tasks as well as on the class of attributes (semantic or physical) toward which encoding is biased. The possibility of equal retention on the basis of different attributes also makes specification of encoding an important issue. Evidence that is independent of overall differences in retention must be used to demonstrate that encoding differs among experimental conditions.

\section{REFERENCES}

Battig, W. F., \& Montague, W. E. Category norms for verbal items in 56 categories: $A$ replication and extension of the Connecticut category norms. Journal of Experimental Psychology, 1969, 80(3, Part 2).

Begg, I., \& Paivio, A. Concreteness and imagery in sentence meaning. Journal of Verbal Learning \& Verbal Behavior, $1969,8,821-827$.

Craik, F. I. M. A "levels of analysis" view of memory. In P. Pliner, L. Krames, and T. M. Alloway (Eds.), Communication and affect: Language and thought. New York: Academic Press, 1973.

Ekstrand, B. R., Wallace, W. P., \& Underwood, B. J. A frequency theory of verbal-discrimination learning. Psychological Review, $1966,73,566-578$.

Elias, C. S., \& Perfetti, C. A. Encoding task and recognition memory: The importance of semantic encoding. Journal of Experimental Psychology, 1973, 99, 151-156.

Jacoby, L. L., \& Goolkasian, P. Semantic versus acoustic coding: Retention and conditions of organization. Journal of Verbal Learning \& Verbal Behavior, 1973, 12, 324-333.

Kolers, P. K. R emember operations. Memory \& Cognition, 1973, $1,347-355$.

Light, L. L., \& Carter-Sobell, L. Effects of changed semantic context on recognition memory. Journal of Verbal Learning \& Verbal Behavior, 1970, 9, 1-11.

Sachs, J. S. Recognition memory for sytactic and semantic aspects of connected discourse. Perception \& Psychophysics, $1967,2,437-442$.

Tulving. E., \& Thomson, D. W. Retrieval processes in recognition memory: Effects of associative context. Journal of Experimental Psychology, 1971, 87, 116-125.

Underwood, B. J. Attributes of memory. Psychological Review, $1969,76,559-573$.

Walter, $D$. A. The effect of sentence context on the stability of phonemic and semantic memory dimensions. Journal of Verbal Learning \& Verbal Behavior, 1973, 12, 185-192.

Wickens, D. D. Encoding categories or words: Am empirical approach to meaning. Psychological R eview, 1970, 77, 1-15.

(R eceived for publication March 18, 1974, revision received May $31,1974$. 